\title{
Convergence of the $q$-Stancu-Szász-Beta type operators
}

\section{Ülkü Dinlemez*}

"Correspondence: ulku@gazi.edu.tr Department of Mathematics,

Faculty of Science, Gazi University, Teknikokullar, Ankara 06500, Turkey

\begin{abstract}
In this paper, we study on q-Stancu-Szász-Beta type operators. We give these operators convergence properties and obtain a weighted approximation theorem in the interval $[0, \infty)$.
\end{abstract}

MSC: $41 \mathrm{~A} 25 ; 41 \mathrm{~A} 36$

Keywords: q-Stancu type operators; Szász-Beta type operators; weighted approximation

\section{Introduction}

In [1], Mahmudov constructed $q$-Szász operators and obtained rate of global convergence in the frame of weighted spaces and a Voronovskaja type theorem for these operators. In [2], Gupta and Mahmudov studied on the $q$-analog of the Szász-Beta type operators. In [3], Yüksel and Dinlemez gave a Voronovskaja type theorem for $q$-analog of a certain family Szász-Beta type operators. In [4], Govil and Gupta introduced the $q$-analog of certain Beta-Szász-Stancu operators. They estimated the moments and established direct results in terms of modulus of continuity and an asymptotic formula for the $q$-operators. In [5-14], interesting generalization about $q$-calculus were given. Our aims are to give approximation properties and a weighted approximation theorem for $q$-Stancu-Szász-Beta type operators. We use without further explanation the basic notations and formulas, from the theory of $q$-calculus as set out in [15-19]. Let $A>0$ and $f$ be a real valued continuous function defined on the interval $[0, \infty)$. For $0<q \leq 1, q$-Stancu-Szász-Beta type operators are defined as

$$
B_{n, q}^{(\alpha, \beta)}(f, x)=\sum_{k=0}^{\infty} s_{n, k}^{q}(x) \int_{0}^{\infty / A} b_{n, k}^{q}(t) f\left(\frac{[n]_{q} t+\alpha}{[n]_{q}+\beta}\right) d_{q} t
$$

where

$$
s_{n, k}^{q}(x)=\left([n]_{q} x\right)^{k} \frac{e^{-[n]_{q} x}}{[k]_{q} !}
$$

and

$$
b_{n, k}^{q}(x)=\frac{q^{k^{2}} x^{k}}{B_{q}(k+1, n)(1+x)_{q}^{n+k+1}} .
$$

๑) 2014 Dinlemez; licensee Springer. This is an Open Access article distributed under the terms of the Creative Commons Attribution License (http://creativecommons.org/licenses/by/2.0), which permits unrestricted use, distribution, and reproduction in any medium, provided the original work is properly cited. 
If we write $q=1$ and $\alpha=\beta=0$ in (1.1), then the operators $B_{n, q}^{(\alpha, \beta)}(f, x)$ are reduced to SzászBeta type operators studied in [20-23].

\section{Auxiliary results}

For the sake of brevity, the notation $F_{s}^{q}(n)=\prod_{i=1}^{s}[n-i]_{q}$ and $G_{\beta}^{q}(n)=\left([n]_{q}+\beta\right)$ will be used throughout the article. Now we are ready to give the following lemma for the Korovkin test functions.

Lemma 1 Let $e_{m}(t)=t^{m}, m=0,1,2$, we get

(i) $B_{n, q}^{(\alpha, \beta)}\left(e_{0}, x\right)=1$,

(ii) $\quad B_{n, q}^{(\alpha, \beta)}\left(e_{1}, x\right)=\frac{[n]_{q}^{2} x}{q^{2} G_{\beta}^{q}(n) F_{1}^{q}(n)}+\frac{[n]_{q}}{q G_{\beta}^{q}(n) F_{1}^{q}(n)}+\frac{\alpha}{G_{\beta}^{q}(n)}$,

(iii) $\quad B_{n, q}^{(\alpha, \beta)}\left(e_{2}, x\right)=\frac{[n]_{q}^{4} x^{2}}{q^{6} G_{\beta}^{q}(n)^{2} F_{2}^{q}(n)}+\left\{\frac{[n]_{q}^{3}}{q^{5} G_{\beta}^{q}(n)^{2} F_{2}^{q}(n)}\right.$

$$
\begin{aligned}
& \left.+\frac{\left(1+[2]_{q}\right)[n]_{q}^{3}}{q^{4} G_{\beta}^{q}(n)^{2} F_{2}^{q}(n)}+\frac{2 \alpha[n]_{q}^{2}}{q^{2} G_{\beta}^{q}(n)^{2} F_{1}^{q}(n)}\right\} x \\
& +\frac{[2]_{q}[n]_{q}^{2}}{q^{3} G_{\beta}^{q}(n)^{2} F_{2}^{q}(n)}+\frac{2 \alpha[n]_{q}}{q G_{\beta}^{q}(n)^{2} F_{1}^{q}(n)}+\frac{\alpha^{2}}{G_{\beta}^{q}(n)^{2}} .
\end{aligned}
$$

Proof Using the $q$-Gamma and $q$-Beta functions in $[15,24]$, we obtain the following equality:

$$
\begin{aligned}
q^{k^{2}} & \int_{0}^{\infty / A} \frac{1}{B(k+1, n)} \frac{t^{k+m}}{(1+t)_{q}^{n+k+1}} d_{q} t \\
= & \frac{[m+k]_{q} ![n-m-1]_{q} ! q^{\left\{2 k^{2}-(k+m)(k+m+1)\right\} / 2}}{[k]_{q} ![n-1]_{q} !} .
\end{aligned}
$$

Then, using (2.1), for $m=0$, we get

$$
\begin{aligned}
B_{n, q}^{(\alpha, \beta)}\left(e_{0}, x\right) & =e^{-[n]_{q} x} \sum_{k=0}^{\infty} \frac{\left([n]_{q} x\right)^{k}}{[k]_{q} !} q^{k(k-1) / 2} \\
& =e^{-[n]_{q} x} E_{q}^{[n]_{q} x}=1,
\end{aligned}
$$

and the proof of (i) is finished. With a direct computation, we obtain (ii) as follows:

$$
\begin{aligned}
B_{n, q}^{(\alpha, \beta)}\left(e_{1}, x\right)= & \frac{[n]_{q}}{G_{\beta}^{q}(n) F_{1}^{q}(n)} \sum_{k=1}^{\infty} \frac{\left([n]_{q} x\right)^{k}}{[k-1]_{q} !} q^{k(k-3)-2 / 2} e^{-[n]_{q} x} \\
& +\frac{[n]_{q}}{G_{\beta}^{q}(n) F_{1}^{q}(n)} \sum_{k=0}^{\infty} \frac{\left([n]_{q} x\right)^{k}}{[k]_{q} !} q^{k(k-1)-2 / 2} e^{-[n]_{q} x} \\
& +\frac{\alpha}{G_{\beta}^{q}(n)} \sum_{k=0}^{\infty} \frac{\left([n]_{q} x\right)^{k}}{[k]_{q} !} q^{k(k-1) / 2} e^{-[n]_{q} x}
\end{aligned}
$$


Dinlemez Journal of Inequalities and Applications 2014, 2014:354

Page 3 of 8

http://www.journalofinequalitiesandapplications.com/content/2014/1/354

$$
\begin{aligned}
= & \frac{[n]_{q}^{2} x}{q^{2} G_{\beta}^{q}(n) F_{1}^{q}(n)} E_{q}^{[n]_{q} x} e^{-[n]_{q} x}+\frac{[n]_{q}}{q G_{\beta}^{q}(n) F_{1}^{q}(n)} E_{q}^{[n]_{q} x} e^{-[n]_{q} x} \\
& +\frac{\alpha}{G_{\beta}^{q}(n)} E_{q}^{[n]_{q} x} e^{-[n]_{q} x} \\
= & \frac{[n]_{q}^{2} x}{q^{2} G_{\beta}^{q}(n) F_{1}^{q}(n)}+\frac{[n]_{q}}{q G_{\beta}^{q}(n) F_{1}^{q}(n)}+\frac{\alpha}{G_{\beta}^{q}(n)} .
\end{aligned}
$$

Using the equality

$$
[n]_{q}=[s]_{q}+q^{s}[n-s]_{q}, \quad 0 \leq s \leq n,
$$

we get

$$
\begin{aligned}
B_{n, q}^{(\alpha, \beta)}\left(e_{2}, x\right)= & \frac{[n]_{q}^{4} x^{2}}{q^{6} G_{\beta}^{q}(n)^{2} F_{2}^{q}(n)} \\
& +\left\{\frac{[n]_{q}^{3}}{q^{5} G_{\beta}^{q}(n)^{2} F_{2}^{q}(n)}+\frac{\left(1+[2]_{q}\right)[n]_{q}^{3}}{q^{4} G_{\beta}^{q}(n)^{2} F_{2}^{q}(n)}+\frac{2 \alpha[n]_{q}^{2}}{q^{2} G_{\beta}^{q}(n)^{2} F_{1}^{q}(n)}\right\} x \\
& +\frac{[2]_{q}[n]_{q}^{2}}{q^{3} G_{\beta}^{q}(n)^{2} F_{2}^{q}(n)}+\frac{2 \alpha[n]_{q}}{q G_{\beta}^{q}(n)^{2} F_{1}^{q}(n)}+\frac{\alpha^{2}}{G_{\beta}^{q}(n)^{2}},
\end{aligned}
$$

and so we have the proof of (iii).

To obtain our main results we need to compute the second moment.

Lemma 2 Let $q \in(0,1)$ and $n>2$. Then we have the following inequality:

$$
B_{n, q}^{(\alpha, \beta)}\left((t-x)^{2}, x\right) \leq\left(\frac{2\left(1-q^{4}\right)}{q^{6}}+\frac{164(\alpha+\beta+1)^{2}[n]_{q}}{q^{6} F_{2}^{q}(n)}\right) x(x+1)+\frac{6(\alpha+1)^{2}}{q^{3} G_{\beta}^{q}(n)}
$$

Proof From the linearity of the $B_{n, q}^{(\alpha, \beta)}$ operators and Lemma 1, we write the second moment as

$$
\begin{aligned}
B_{n, q}^{(\alpha, \beta)}\left((t-x)^{2}, x\right) \\
=\left\{\frac{[n]_{q}^{4}}{q^{6} G_{\beta}^{q}(n)^{2} F_{2}^{q}(n)}-\frac{2[n]_{q}^{2}}{q^{2} G_{\beta}^{q}(n) F_{1}^{q}(n)}+1\right\} x^{2} \\
+\left\{\frac{\left\{1+\left(1+[2]_{q}\right) q\right\}[n]_{q}^{3}}{q^{5} G_{\beta}^{q}(n)^{2} F_{2}^{q}(n)}+\frac{2 \alpha[n]_{q}^{2}}{q^{2} G_{\beta}^{q}(n)^{2} F_{1}^{q}(n)}-\frac{2[n]_{q}}{q G_{\beta}^{q}(n) F_{1}^{q}(n)}-\frac{2 \alpha}{G_{\beta}^{q}(n)}\right\} x \\
+\frac{[2]_{q}[n]_{q}^{2}}{q^{3} G_{\beta}^{q}(n)^{2} F_{2}^{q}(n)}+\frac{2 \alpha[n]_{q}}{q G_{\beta}^{q}(n)^{2} F_{1}^{q}(n)}+\frac{\alpha^{2}}{G_{\beta}^{q}(n)^{2}} \\
\leq\left\{\frac{[n]_{q}^{4}}{q^{6} G_{\beta}^{q}(n)^{2} F_{2}^{q}(n)}-\frac{2[n]_{q}^{2}}{q^{2} G_{\beta}^{q}(n) F_{1}^{q}(n)}+1+\frac{\left\{1+\left(1+[2]_{q}\right) q\right\}[n]_{q}^{3}}{q^{5} G_{\beta}^{q}(n)^{2} F_{2}^{q}(n)}\right. \\
\left.+\frac{2 \alpha[n]_{q}^{2}}{q^{2} G_{\beta}^{q}(n)^{2} F_{1}^{q}(n)}\right\} x(x+1)+\frac{[2]_{q}[n]_{q}^{2}}{q^{3} G_{\beta}^{q}(n)^{2} F_{2}^{q}(n)}+\frac{2 \alpha[n]_{q}}{q G_{\beta}^{q}(n)^{2} F_{1}^{q}(n)}+\frac{\alpha^{2}}{G_{\beta}^{q}(n)^{2}}
\end{aligned}
$$




$$
\begin{aligned}
\leq & \left\{\frac{[n]_{q}^{4}\left(1+q^{6}\right)-2 q^{4}[n-2]_{q}^{4}+2 \beta q^{6}[n]_{q}[n-1]_{q}[n-2]_{q}}{q^{6} G_{\beta}^{q}(n)^{2} F_{2}^{q}(n)}\right. \\
& \left.+\frac{\left(q+q^{2}+[2]_{q} q^{2}\right)[n]_{q}^{3}}{q^{6} G_{\beta}^{q}(n)^{2} F_{2}^{q}(n)}+\frac{q^{6} \beta^{2}[n-1]_{q}[n-2]_{q}}{q^{6} G_{\beta}^{q}(n)^{2} F_{2}^{q}(n)}+\frac{2 \alpha q^{4}[n]_{q}^{2}[n-2]_{q}}{q^{6} G_{\beta}^{q}(n)^{2} F_{2}^{q}(n)}\right\} x(x+1) \\
& +\frac{\left\{[2]_{q}+2 \alpha q^{2}+\alpha^{2} q^{3}\right\}[n]_{q}}{q^{3} G_{\beta}^{q}(n) F_{2}^{q}(n)} .
\end{aligned}
$$

From (2.2), we have

$$
\begin{aligned}
B_{n, q}^{(\alpha, \beta)}\left((t-x)^{2}, x\right) \\
\leq\left\{\frac{[n-2]_{q}^{4}\left(q^{14}+q^{8}-2 q^{4}\right)}{q^{6} G_{\beta}^{q}(n)^{2} F_{2}^{q}(n)}\right. \\
\quad+\frac{\left(1+q^{6}\right)\left\{4[2]_{q} q^{6}[n-2]_{q}^{3}+6[2]_{q}^{2} q^{4}[n-2]_{q}^{2}+4[2]_{q}^{3} q^{2}[n-2]_{q}+[2]_{q}^{4}\right\}}{q^{6} G_{\beta}^{q}(n)^{2} F_{2}^{q}(n)} \\
\left.\quad+\frac{\left(q+q^{2}+[2]_{q} q^{2}+2 \beta q^{6}+2 \alpha q^{4}\right)[n]_{q}^{3}+\beta^{2} q^{6}[n]_{q}^{2}}{q^{6} G_{\beta}^{q}(n)^{2} F_{2}^{q}(n)}\right\} x(x+1) \\
\quad+\frac{\left([2]_{q}+q^{2}\right)\left([2]_{q}+2 \alpha q^{2}+\alpha^{2} q^{3}\right)}{q^{3} G_{\beta}^{q}(n) F_{1}^{q}(n)} \\
\leq\left(\frac{2\left(1-q^{4}\right)}{q^{6}}+\frac{164(\alpha+\beta+1)^{2}[n]_{q}}{q^{6} F_{2}^{q}(n)}\right) x(x+1)+\frac{6(\alpha+1)^{2}}{q^{3} G_{\beta}^{q}(n)} .
\end{aligned}
$$

And the proof of Lemma 2 is now finished.

\section{Direct estimates}

Now in our considerations, $C_{B}[0, \infty)$ denotes the set of all bounded-continuous functions from $[0, \infty)$ to $\mathbb{R} . C_{B}[0, \infty)$ is a normed space with the norm $\|f\|_{B}=\sup \{|f(x)|: x \in[0, \infty)\}$. We denote the first modulus of continuity on the finite interval $[0, b], b>0$,

$$
\omega_{[0, b]}(f, \delta)=\sup _{0<h \leq \delta, x \in[0, b]}|f(x+h)-f(x)| .
$$

The Peetre $K$-functional is defined by

$$
K_{2}(f, \delta)=\inf \left\{\|f-g\|_{B}+\delta\left\|g^{\prime \prime}\right\|_{B}: g \in W_{\infty}^{2}\right\}, \quad \delta>0,
$$

where $W_{\infty}^{2}=\left\{g \in C_{B}[0, \infty): g^{\prime}, g^{\prime \prime} \in C_{B}[0, \infty)\right\}$. By Theorem 2.4 in [25], p.177, there exists a positive constant $C$ such that

$$
K_{2}(f, \delta) \leq C \omega_{2}(f, \sqrt{\delta})
$$

where

$$
\omega_{2}(f, \sqrt{\delta})=\sup _{0<h \leq \sqrt{\delta}} \sup _{x \in[0, \infty)}|f(x+2 h)-2 f(x+h)-f(x)| .
$$

Gadzhiev proved the weighted Korovkin-type theorems in [26]. We give the Gadzhiev results in weighted spaces. Let $\rho(x)=1+x^{2}$ and the weighted spaces $C_{\rho}[0, \infty)$ denote 
the space of all continuous functions $f$, satisfying $|f(x)| \leq M_{f} \rho(x)$, where $M_{f}$ is a constant depending only on $f . C_{\rho}[0, \infty)$ is a normed space with the norm $\|f\|_{\rho}=\sup \left\{\frac{|f(x)|}{\rho(x)}\right.$ : $\left.x \in \mathbb{R}^{+} \cup\{0\}\right\}$ and $C_{\rho}^{*}[0, \infty)$ denotes the subspace of all functions $f \in C_{\rho}[0, \infty)$ for which $\lim _{|x| \rightarrow \infty} \frac{|f(x)|}{\rho(x)}$ exists finitely.

Thus we are ready to give direct results. The following lemma is routine and its proof is omitted.

\section{Lemma 3 Let}

$$
\bar{B}_{n, q}^{(\alpha, \beta)}(f, x)=B_{n, q}^{(\alpha, \beta)}(f, x)-f\left(D_{n, q}^{(\alpha, \beta)}(x)\right)+f(x) .
$$

Then the following assertions hold for the operators (3.3):

$$
\begin{aligned}
\text { (i) } & \bar{B}_{n, q}^{(\alpha, \beta)}(1, x)=1, \\
\text { (ii) } & \bar{B}_{n, q}^{(\alpha, \beta)}(t, x)=x, \\
\text { (iii) } & \bar{B}_{n, q}^{(\alpha, \beta)}(t-x, x)=0,
\end{aligned}
$$

where $D_{n, q}^{(\alpha, \beta)}(x)=\frac{[n]_{q}^{2} x}{q^{2} G_{\beta}^{q}(n) F_{1}^{q}(n)}+\frac{[n]_{q}}{q G_{\beta}^{q}(n) F_{1}^{q}(n)}+\frac{\alpha}{G_{\beta}^{q}(n)}$.

Lemma 4 Let $q \in(0,1)$ and $n>2$. Then for every $x \in[0, \infty)$ and $f^{\prime \prime} \in C_{B}[0, \infty)$, we have the inequality

$$
\left|\bar{B}_{n, q}^{(\alpha, \beta)}(f, x)-f(x)\right| \leq \delta_{n, q}^{(\alpha, \beta)}(x)\left\|f^{\prime \prime}\right\|_{B^{\prime}}
$$

where $\delta_{n, q}^{(\alpha, \beta)}(x)=\left(\frac{2\left(1-q^{4}\right)}{q^{6}}+\frac{263(\alpha+\beta+1)^{2}}{q^{6} F_{1}^{q}(n)}\right) x(x+1)+\frac{5(\alpha+1)^{2}}{q^{3} G_{\beta}^{q}(n)}$.

Proof Using Taylor's expansion

$$
f(t)=f(x)+(t-x) f^{\prime}(x)+\int_{x}^{t}(t-u) f^{\prime \prime}(u) d u
$$

and Lemma 3, we obtain

$$
\bar{B}_{n, q}^{(\alpha, \beta)}(f, x)-f(x)=\bar{B}_{n, q}^{(\alpha, \beta)}\left(\int_{x}^{t}(t-u) f^{\prime \prime}(u) d u, x\right) .
$$

Then, using Lemma 1 and the inequality

$$
\left|\int_{x}^{t}(t-u) f^{\prime \prime}(u) d u\right| \leq\left\|f^{\prime \prime}\right\|_{B} \frac{(t-x)^{2}}{2}
$$

we get

$$
\begin{aligned}
& \left|\bar{B}_{n, q}^{(\alpha, \beta)}(f, x)-f(x)\right| \\
& \quad \leq\left|B_{n, q}^{(\alpha, \beta)}\left(\int_{x}^{t}(t-u) f^{\prime \prime}(u) d u, x\right)-\int_{x}^{D_{n, q}^{(\alpha, \beta)}(x)}\left\{D_{n, q}^{(\alpha, \beta)}(x)-u\right\} f^{\prime \prime}(u) d u\right|
\end{aligned}
$$




$$
\begin{aligned}
\leq & \frac{\left\|f^{\prime \prime}\right\|_{B}}{2}\left\{\left(\frac{2\left(1-q^{4}\right)}{q^{6}}+\frac{164(\alpha+\beta+1)^{2}[n]_{q}}{q^{6} F_{2}^{q}(n)}+\left(\frac{[n]_{q}^{2}}{q^{2} G_{\beta}^{q}(n) F_{1}^{q}(n)}-1\right)^{2}\right.\right. \\
& \left.+\frac{2[n]_{q}^{3}}{q^{3} G_{\beta}^{q}(n)^{2} F_{1}^{q}(n)^{2}}+\frac{2[n]_{q}^{2} \alpha}{q^{2} G_{\beta}^{q}(n)^{2} F_{1}^{q}(n)}\right) x(x+1)+\left(\frac{[n]_{q}+\alpha q[n-1]_{q}}{q G_{\beta}^{q}(n) F_{1}^{q}(n)}\right)^{2} \\
& \left.+\frac{6(\alpha+1)^{2}}{q^{3} G_{\beta}^{q}(n) F_{1}^{q}(n)}\right\} \\
\leq & \frac{\left\|f^{\prime \prime}\right\|_{B}}{2}\left\{\left(\frac{4\left(1-q^{4}\right)}{q^{6}}+\frac{526(\alpha+\beta+1)^{2}}{q^{6} F_{1}^{q}(n)}\right) x(x+1)+\frac{10(\alpha+1)^{2}}{q^{3} G_{\beta}^{q}(n)}\right\} .
\end{aligned}
$$

And the proof of the Lemma 4 is now completed.

Theorem 1 Let $\left(q_{n}\right) \subset(0,1)$ a sequence such that $q_{n} \rightarrow 1$ as $n \rightarrow \infty$. Then for every $n>2$, $x \in[0, \infty)$ and $f \in C_{B}[0, \infty)$, we have the inequality

$$
\left|B_{n, q_{n}}^{(\alpha, \beta)}(f, x)-f(x)\right| \leq 2 M \omega_{2}\left(f, \sqrt{\delta_{n, q_{n}}^{(\alpha, \beta)}(x)}\right)+w\left(f, \eta_{n, q_{n}}^{(\alpha, \beta)}(x)\right)
$$

where $\eta_{n, q_{n}}^{(\alpha, \beta)}(x)=\left(\frac{[n]_{q_{n}}^{2}}{q_{n}^{2} G_{\beta}^{q_{n}}(n) F_{1}^{q_{n}}(n)}-1\right) x+\frac{[n]_{q_{n}}}{q_{n} G_{\beta}^{q_{n}}(n) F_{1}^{q_{n}}(n)}+\frac{\alpha}{G_{\beta}^{q_{n}}(n)}$.

Proof Using (3.3) for any $g \in W_{\infty}^{2}$, we obtain the following inequality:

$$
\begin{aligned}
\left|B_{n, q_{n}}^{(\alpha, \beta)}(f, x)-f(x)\right| \leq & \left|\bar{B}_{n, q_{n}}^{(\alpha, \beta)}(f-g, x)-(f-g)(x)+\bar{B}_{n, q_{n}}^{(\alpha, \beta)}(g, x)-g(x)\right| \\
& +\left|f\left(\frac{[n]_{q_{n}}^{2}}{q_{n}^{2} G_{\beta}^{q_{n}}(n) F_{1}^{q_{n}}(n)} x+\frac{[n]_{q_{n}}}{q_{n} G_{\beta}^{q_{n}}(n) F_{1}^{q_{n}}(n)}+\frac{\alpha}{G_{\beta}^{q_{n}}(n)}\right)-f(x)\right| .
\end{aligned}
$$

From Lemma 4, we get

$$
\begin{aligned}
\left|B_{n, q_{n}}^{(\alpha, \beta)}(f, x)-f(x)\right| \leq & 2\|f-g\|_{B}+\delta_{n, q_{n}}^{(\alpha, \beta)}(x)\left\|g^{\prime \prime}\right\| \\
& +\left|f\left(\frac{[n]_{q_{n}}^{2}}{q_{n}^{2} G_{\beta}^{q_{n}}(n) F_{1}^{q_{n}}(n)} x+\frac{[n]_{q_{n}}}{q_{n} G_{\beta}^{q_{n}}(n) F_{1}^{q_{n}}(n)}+\frac{\alpha}{G_{\beta}^{q_{n}}(n)}\right)-f(x)\right| .
\end{aligned}
$$

By using equality (3.1) we have

$$
\left|B_{n, q_{n}}^{(\alpha, \beta)}(f, x)-f(x)\right| \leq 2\|f-g\|_{B}+\delta_{n, q_{n}}^{(\alpha, \beta)}(x)\left\|g^{\prime \prime}\right\|_{B}+w\left(f, \eta_{n, q_{n}}^{(\alpha, \beta)}(x)\right) .
$$

Taking the infimum over $g \in W_{\infty}^{2}$ on the right-hand side of the above inequality and using the inequality (3.2), we get the desired result.

Theorem 2 Let $\left(q_{n}\right) \subset(0,1)$ a sequence such that $q_{n} \rightarrow 1$ as $n \rightarrow \infty$. Then $f \in C_{\rho}^{*}[0, \infty)$, and we have

$$
\lim _{n \rightarrow \infty}\left\|B_{n, q_{n}}^{(\alpha, \beta)}(f)-f\right\|_{\rho}=0
$$

Proof From Lemma 1, it is obvious that $\left\|B_{n, q_{n}}^{(\alpha, \beta)}\left(e_{0}\right)-e_{0}\right\|_{\rho}=0$. Since $\mid \frac{[n]_{q_{n}}^{2}}{q_{n}^{2} G_{\beta}^{q_{n}}(n) F_{1}^{q_{n}}(n)} x+$ $\frac{[n]_{q_{n}}}{q_{n} G_{\beta}^{q^{q n}}(n) F_{1}^{q_{n}}(n)}+\frac{\alpha}{G_{\beta}^{q n}(n)}-x \mid \leq(x+1) o(1)$ and $\frac{x+1}{1+x^{2}}$ is positive and bounded from above for 
each $x \geq 0$, we obtain

$$
\left\|B_{n, q_{n}}^{(\alpha, \beta)}\left(e_{1}\right)-e_{1}\right\|_{\rho} \leq \frac{x+1}{1+x^{2}} o(1) .
$$

And then $\lim _{n \rightarrow \infty}\left\|B_{n, q_{n}}^{(\alpha, \beta)}\left(e_{1}\right)-e_{1}\right\|_{\rho}=0$

Similarly for every $n>2$, we write

$$
\begin{aligned}
\left\|B_{n, q_{n}}^{(\alpha, \beta)}\left(e_{2}\right)-e_{2}\right\|_{\rho}= & \sup _{x \in[0, \infty)}\left\{\frac{\mid\left(\frac{[n]_{q_{n}}^{4}}{q_{n}^{6} G_{\beta}^{q_{n}}(n)^{2} F_{2}^{q_{n}}(n)}-1\right) x^{2}}{1+x^{2}}\right. \\
& +\frac{\left\{\frac{\left(1+\left(1+[2]_{q_{n}}\right) q_{n}\right)[n]_{q_{n}}^{3}+2 \alpha q^{2}[n]_{q_{n}}^{2}[n-1]_{q_{n}}}{q_{n}^{5} G_{\beta}^{q_{n}}(n)^{2} F_{2}^{q_{n}}(n)}\right\} x+\frac{[2]_{q_{n}}[n]_{q_{n}}^{2}}{q_{n}^{3} G_{\beta}^{q_{n}}(n)^{2} F_{2}^{q_{n}}(n)}}{1+x^{2}} \\
& \left.+\frac{\frac{+2 \alpha q_{n}^{2}[n]_{q_{n}}[n-2]_{q_{n}}}{q_{n}^{3} G_{\beta}^{q_{n}}(n)^{2} F_{2}^{q_{n}}(n)}+\frac{\alpha^{2}}{G_{\beta}^{q_{n}}(n)^{2}} \mid}{1+x^{2}}\right\} \\
\leq & \sup _{x \in[0, \infty)} \frac{1+x+x^{2}}{1+x^{2}} o(1),
\end{aligned}
$$

we get $\lim _{n \rightarrow \infty}\left\|B_{n, q_{n}}^{(\alpha, \beta)}\left(e_{2}\right)-e_{2}\right\|_{\rho}=0$. Thus, from AD Gadzhiev's theorem in [26], we obtain the desired result of Theorem 2 .

\section{Competing interests}

The author declares to have no competing interests.

\section{Acknowledgements}

The author would like thank the referee for many helpful comments.

Received: 2 April 2014 Accepted: 27 August 2014 Published: 24 September 2014

\section{References}

1. Mahmudov, Nl: q-Szász operators which preserve $x^{2}$. Math. Slovaca 63, 1059 (2013)

2. Gupta, V, Mahmudov, NI: Approximation properties of the $q$-Szasz-Mirakjan-Beta operators. Indian J. Ind. Appl. Math. 3, $41(2012)$

3. Govil, NK, Gupta, V: q-Beta-Szász-Stancu operators. Adv. Stud. Contemp. Math. 22, 117 (2012)

4. Yüksel, I, Dinlemez, Ü: On the approximation by the q-Szász-Beta type operators. Appl. Math. Comput. 235, 555 (2014)

5. Dinlemez, Ü, Yüksel, I, Altın, B: A note on the approximation by the $q$-hybrid summation integral type operators. Taiwan. J. Math. 18, 781 (2014)

6. Doğru, O, Gupta, V: Monotonicity and the asymptotic estimate of Bleimann Butzer and Hahn operators based on q-integers. Georgian Math. J. 12, 415 (2005)

7. Doğru, O, Gupta, V: Korovkin-type approximation properties of bivariate $q$-Meyer-König and Zeller operators. Calcolo 43, $51(2006)$

8. Gupta, V, Heping, W: The rate of convergence of $q$-Durrmeyer operators for $0<q<1$. Math. Methods Appl. Sci. 31, $1946(2008)$

9. Gupta, V, Aral, A: Convergence of the q-analogue of Szász-Beta operators. Appl. Math. Comput. 216, 374 (2010)

10. Gupta, V, Karsli, H: Some approximation properties by q-Szász-Mirakyan-Baskakov-Stancu operators. Lobachevskii J. Math. 33, 175 (2012)

11. Lupaş, A: A q-analogue of the Bernstein operator. In: Seminar on Numerical and Statistical Calculus, pp. 85-92 (1987)

12. Phillips, GM: Bernstein polynomials based on the $q$-integers. Ann. Numer. Math. 4, 511 (1997)

13. Yüksel, I: Approximation by q-Phillips operators. Hacet. J. Math. Stat. 40, 191 (2011)

14. Yüksel, l: Approximation by q-Baskakov-Schurer-Szász type operators. AIP Conf. Proc. 1558, 1136 (2013). doi:10.1063/1.4825708

15. De Sole, A, Kac, VG: On integral representations of q-gamma and q-Beta functions. Atti Accad. Naz. Lincei, Rend. Lincei, Mat. Appl. 16, 11 (2005)

16. Gupta, V, Agarwal, RP: Convergence Estimates in Approximation Theory. Springer, Cham (2014). ISBN:978-3-319-02764-7

17. Jackson, FH: On q-definite integrals. Q. J. Pure Appl. Math. 41, 193 (1910) 
18. Kac, VG, Cheung, P: Quantum Calculus. Universitext. Springer, New York (2002)

19. Koelink, HT, Koornwinder, TH: $q$-Special functions, a tutorial. In: Deformation Theory and Quantum Groups with Applications to Mathematical Physics (Amherst, MA, 1990). Contemp. Math., vol. 134, pp. 141-142. Am. Math. Soc., Providence (1992)

20. Deo, N: Direct result on the Durrmeyer variant of Beta operators. Southeast Asian Bull. Math. 32, 283 (2008)

21. Deo, N: Direct result on exponential-type operators. Appl. Math. Comput. 204, 109 (2008)

22. Gupta, V, Srivastava, GS, Sahai, A: On simultaneous approximation by Szász-Beta operators. Soochow J. Math. 21, 1 (1995)

23. Jung, HS, Deo, N, Dhamija, M: Pointwise approximation by Bernstein type operators in mobile interval. Appl. Math. Comput. 214, 683 (2014)

24. Aral, A, Gupta, V, Agarwal, RP: Applications of q-Calculus in Operator Theory. Springer, New York (2013)

25. De Vore, RA, Lorentz, GG: Constructive Approximation. Springer, Berlin (1993)

26. Gadzhiev, AD: Theorems of the type of P. P. Korovkin type theorems. Mat. Zametki 20, 781 (1976). English Translation, Math. Notes 20, 996 (1976)

doi:10.1186/1029-242X-2014-354

Cite this article as: Dinlemez: Convergence of the $q$-Stancu-Szász-Beta type operators. Journal of Inequalities and Applications 2014 2014:354.

\section{Submit your manuscript to a SpringerOpen ${ }^{\circ}$ journal and benefit from:}

- Convenient online submission

- Rigorous peer review

- Immediate publication on acceptance

- Open access: articles freely available online

- High visibility within the field

- Retaining the copyright to your article 